\title{
Performance Assessment of a New Electromyography-based Neuromuscular Monitor and Subjective Discomfort in Unmedicated Volunteers
}

Réka Nemes, Boglárka Diószeghy, Adrienn Pongrácz, Edömér Tassonyi and Béla Fülesdi*

Department of Anesthesiology and Intensive Care, University of Debrecen, 98 Nagyerdei krt, H-4032 Debrecen, Hungary

"Corresponding author: Béla Fülesdi, Department of Anesthesiology and Intensive Care, University of Debrecen, 98 Nagyerdei krt, H-4032 Debrecen, Hungary, Tel: +36303838557; E-mail: fulesdi@med.unideb.hu

Received date: July 20, 2018; Accepted date: July 31, 2018; Published date: August 06, 2018

Copyright: $\odot 2018$ Nemes R, et al. This is an open-access article distributed under the terms of the Creative Commons Attribution License, which permits unrestricted use, distribution, and reproduction in any medium, provided the original author and source are credited.

\section{Abstract}

Objective: The aim of this prospective, unblinded, randomized study was to provide performance data of the prototype of new electromyography-based neuromuscular monitor, the NEAT device (Acacia Designs BV, Amsterdam, the Netherlands) and assess the subjective discomfort associated with neurostimulation in unmedicated healthy volunteers.

Methods: The study enrolled ten male and ten female volunteers. Based on a priori randomization the ulnar nerve of the left or right arms was stimulated in $1 \mathrm{~Hz}$ single twitch and train-of-four modes. Stimulating current intensity was increased from $10 \mathrm{~mA}$ to $60 \mathrm{~mA}$ in $10 \mathrm{~mA}$ steps. Electromyography recordings were performed at the abductor digiti minimi and adductor pollicis muscles via surface electrodes. The volunteers were asked to rate the discomfort in association with neurostimulation on a 0-10 visual analogue scale.

Results: The overall train-of-four ratio was 1.02 (0.98-1.06) \{median and (interquartile range)\}. The abductor digiti minimi muscle required lower stimulating current intensity to evoke maximal stimulation, than the adductor pollicis (30 vs. $50 \mathrm{~mA}, \mathrm{p}<0.001$ ). The overall intracurrent variability of compound muscle action potential amplitudes was $0.42(0.21-0.87) \mathrm{mV}$, that was unaffected by the type of muscle or the stimulating current intensity. Women reported moderately higher visual analogue scale scores than men. The largest recorded difference was 5 (3.75-6) vs. 3 $(3-4), p<0.05$.

Conclusion: The Acacia Designs BV NEAT monitoring device was suitable to deliver neurostimulation, record and analyze the elicited muscle action potentials. The precision of stimulations was acceptable. The volunteers reported the discomfort in association with neurostimulation as tolerable.

Registered at the Hungarian Office for Health Authorization and Administrative Procedures (December $30^{\text {th }}$, 2014). Identifier: 028605-010/2014/OTIG. Registered at clinicaltrials.gov (December 15 ${ }^{\text {th }}$, 2015). Identifier: NCT02630576.

Keywords: Neuromuscular monitoring; Electromyography; Visual analogue scoring scale; Abductor digiti minimi muscle; Adductor pollicis muscle

Abbreviations: AMG: Acceleromyography; ASA: American Society of Anesthesiology; CMAP: Compound Muscle Action Potential; ECG: Electrocardiography; EMG: Electromyography; $\mathrm{I}_{\mathrm{Max}}$ : Current Intensity that Elicited the Highest Compound Muscle Action Potential; $\mathrm{I}_{\mathrm{Th}}$ : Threshold Current Intensity; $\mathrm{I}_{\text {Th-Clinical }}$ : Clinical Threshold Current Intensity; $\mathrm{I}_{\text {Th-EMG: Electromyographical Threshold Current Intensity; }}$ IQR: Interquartile Range; mADM: Abductor Digiti Minimi Muscle; mAP: Adductor Pollicis Muscle; $\mu \mathrm{C}$ : Microcoulombs; mA: Milliampere; msec: Millisecond; $\mathrm{mV}$ : Millivolt; MMG: Mechanomyography; NM: Neuromuscular; PACU Post-Anesthesia Care Unit; PTC: Post-Tetanic Count; SD: Secure Digital; SD: Standard Deviation; ST: Single Twitch; TOF: Train-of-Four; TOFC: Train-ofFour Count; VAS: Visual Analogue Scale

\section{Introduction}

Objective neuromuscular (NM) monitors help optimize the timing and dosing of neuromuscular blocking agents, the timing of safe tracheal extubation at the end of operations, and thus decrease the incidence of residual paralysis and its postoperative consequences [1-4]. Over the last few years, a strong educational effort has been observed, which urged the extended use of NM monitors to improve patient safety and outcomes [2,3,5-8]. In 2009 Kopman proposed that objective neuromuscular monitors be available in any modern anesthetizing location when neuromuscular blocking drugs are administered [9]. In 2016, the Association of Anaesthetists of Great Britain and Ireland prescribed the use of peripheral nerve stimulators whenever NM blocking agent is administered and recommended the use of quantitative monitors [10].

Despite its known limitations, the acceleromyography (AMG) based, portable TOF-Watch series was one of the most frequently used $\mathrm{NM}$ monitor in clinical practice and research. Most recommendations on safe management of NM blockade were based on AMG 
Citation: Nemes R, Diószeghy B, Pongrácz A, Tassonyi E, Fülesdi B (2018) Performance Assessment of a New Electromyography-based Neuromuscular Monitor and Subjective Discomfort in Unmedicated Volunteers. J Anesth Clin Res 9: 848. doi: $10.4172 / 2155-6148.1000848$

Page 2 of 8

investigations $[1,11]$. However, in 2016 the TOF-Watch NM monitors were withdrawn from manufacture. Thus, within a short time, there will be a significant lack of options in the spectrum of stand-alone NM monitors that cannot be overcome by modular original equipment manufacturer NM monitors that are integrated into anesthesia workstations. One reason is that not all anesthesia workstations are equipped with NM modules. In addition, the integrated NM monitors usually are not transported with the patient from the operating room to the postoperative care unit (PACU), which increases the need for additional NM monitors in the PACU. Therefore, there is an increasing need for easy-to-use, reliable, portable NM monitors.

Before the introduction of acceleromyography in the 1980s, mechanomyography (MMG) and electromyography (EMG) were the means to measure neuromuscular function. Although MMG was considered the "gold standard" neuromuscular monitor, it was too bulky for routine use and cumbersome for clinical purposes [2,7], EMG proved to be an excellent alternative. Electromyography measures directly the electrical events of neuromuscular signal transmission, the compound muscle action potentials (CMAP). For this reason, EMG is less susceptible to interference from presynaptic or postsynaptic events, and is likely a better indicator of pure neuromuscular function [2]. Therefore, it is probably the most physiologic and precise method of measuring the synaptic transmission, and thus the degree of NM relaxation [3]. Other practical advantages of EMG over MMG and AMG are that EMG does not require the complete immobilization of the hand, the fixation of the arm in the supine position or the use of a muscle preload. Additionally, the staircase phenomenon does not affect EMG measurements [12], unlike MMG and AMG; therefore, the calibration of the device to determine the supramaximal stimulating current intensity is simpler and shorter. Yet, currently there is no freestanding, hand-held EMG-based NM monitor available in the market. To our knowledge, there are only a couple of anesthesia workstation integrated NM transmission modules that use electromyography or kinemyography (GE Healthcare, Waukesha, WI, USA) [3], or acceleromyography (IntelliVue NMT, Philips, Amsterdam, the
Netherlands; Infinity Trident NMT SmartPod, Dräger, Lübeck, Germany) [3].

The Acacia Designs' prototype monitor (NEAT device, Amsterdam, the Netherlands) is a dedicated electromyography-based NM monitor intended to deliver, record, and analyse CMAPs. The NEAT device is the first prototype of the TetraGraph ${ }^{\mathrm{TM}}$ neuromuscular monitor (Senzime BV, Uppsala, Sweden). The prototype was designed to conduct preclinical investigations. It is battery-powered, uses simple electrocardiography (ECG) electrodes both for stimulation and recording, and registers data on an integrated secure digital (SD) card. The stimulating parameters of the prototype match the parameters of the TetraGraph ${ }^{\mathrm{TM}}$. The aim of the current investigation was to test the prototype that uses the design and electronics intended for the final product, gain performance data and assess the discomfort evoked by neurostimulation in unmedicated, healthy volunteers.

\section{Materials and Methods}

This prospective, unblinded, randomized, single-center study was approved by the Hungarian Office for Health Authorization and Administrative Procedures (028605-010/2014/OTIG) and registered at clinicaltrials.gov (NCT02630576). Data presentation is in accordance with CONSORT 2010 guidelines.

\section{Volunteers}

The study population consisted of normal, healthy volunteers of age 18 years and older, American Society of Anesthesiology (ASA) physical status I. Ten male and ten female volunteers were enrolled in the study (Figure 1). All subjects were required to provide written informed consent prior to inclusion in the study. Exclusion criteria were presence of an underlying neuromuscular disease, use of medications known to interfere with neuromuscular transmission (e.g., antiepileptics, anticholinesterases and magnesium sulphate), presence of renal or hepatic disease, or presence of open sores at the skin sites needed for electrode application.

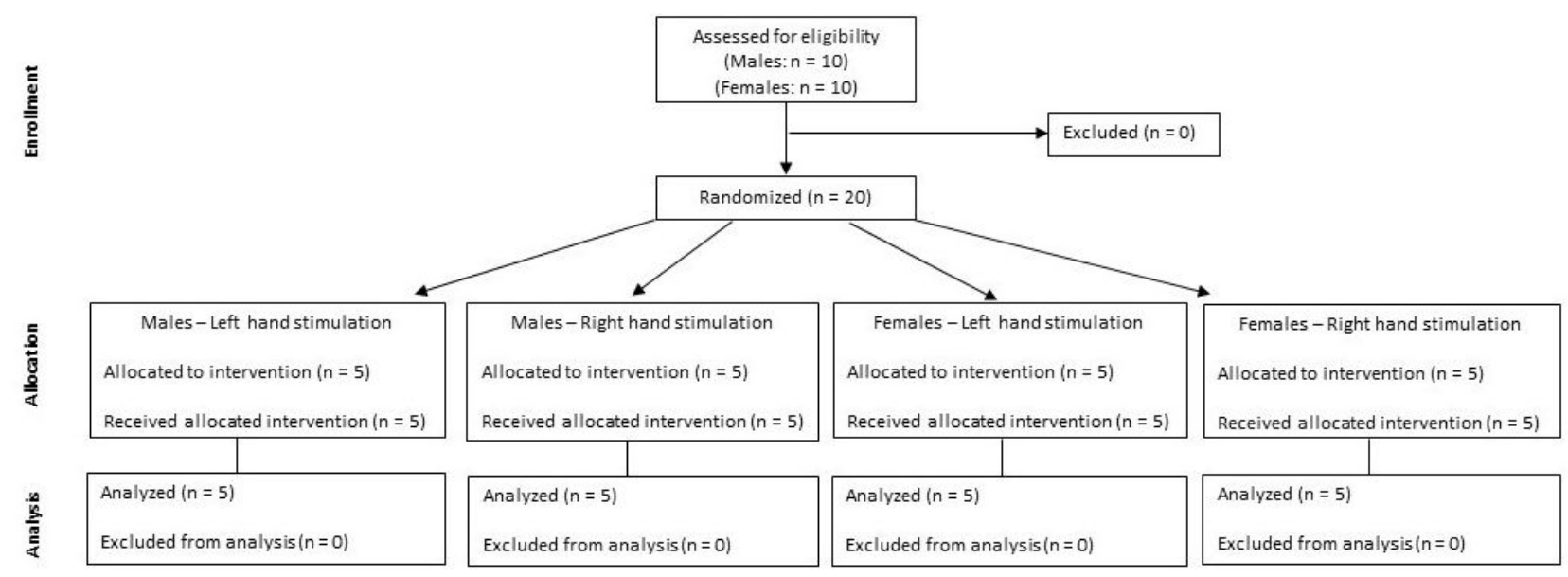

Figure 1: CONSORT study flowchart.

\section{Device specifications}

The NEAT device is the first prototype of a new electromyographybased, battery-powered, portable neuromuscular monitor, whose software was specially developed for research and clinical purposes. It can stimulate the peripheral nerves using parameters and settings currently in use by many peripheral nerve stimulators, and record the evoked muscle response (CMAP). Data is saved onto an SD-card for 
Citation: Nemes R, Diószeghy B, Pongrácz A, Tassonyi E, Fülesdi B (2018) Performance Assessment of a New Electromyography-based Neuromuscular Monitor and Subjective Discomfort in Unmedicated Volunteers. J Anesth Clin Res 9: 848. doi: $10.4172 / 2155-6148.1000848$

Page 3 of 8

subsequent review and analysis. All possible stimulation settings that will be available on the final device were incorporated into this prototype. These available stimulation settings were: variable stimulation current (10-60 mA, in $10 \mathrm{~mA}$ steps); stimulation protocol \{train-of-four (TOF), $1 \mathrm{~Hz}$ single twitch (ST), train-of-four count (TOFC) and post-tetanic count (PTC) $;$; time interval between sequential protocols (every $20 \mathrm{~s}, 1 \mathrm{~min}, 5 \mathrm{~min}, 15 \mathrm{~min}, 60 \mathrm{~min}$ except for the PTC protocol, for which the interval is 3 min minimum between successive stimulations); and stimulus pulse width ( 0.2 or 0.3 $\mathrm{msec}$ ). The prototype was fully battery powered (Varta EZPack XL LiPolymer rechargeable battery, 3.7 V, $2260 \mathrm{mAh}, 8.4 \mathrm{Wh}$, Utrecht, the Netherlands). The NEAT device was used with a double-shielded monitor-to-patient cable and commercially available surface ECG electrodes (3M Red Dot, St. Paul, MN, USA).

\section{Subject preparation and neurostimulation}

Neuromuscular testing was performed at two separate stimulation/ recording sites: ulnar nerve stimulation and abductor digiti minimi muscle (mADM) recording; as well as ulnar nerve stimulation and adductor pollicis muscle (mAP) recording (Figure 2). The side of testing (right or left hand) was determined a priori via envelope randomization, to ensure that 10 volunteers ( 5 male and 5 female) each were tested on the right and 10 on the left hands.

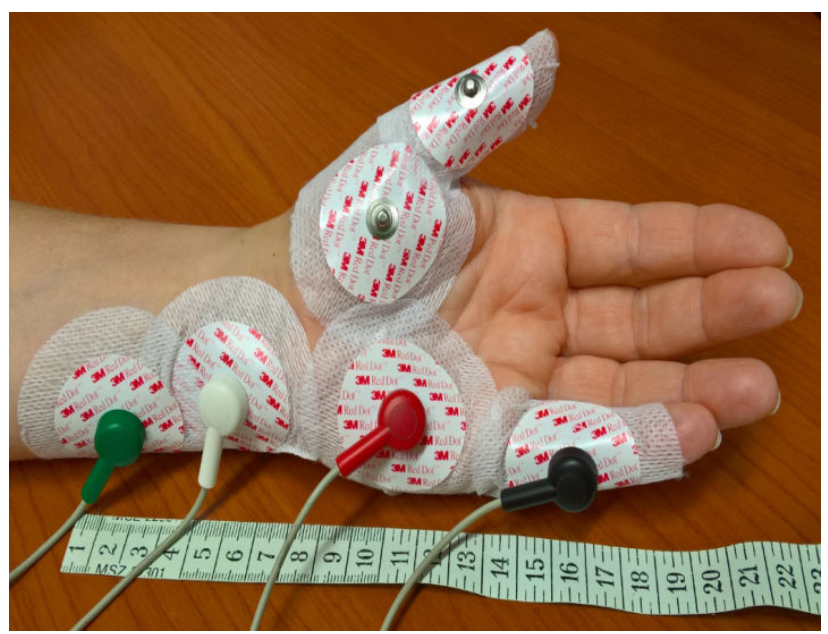

Figure 2: Stimulating and recording electrode placement. Stimulating electrodes are placed along the ulnar nerve. The positive electrode is placed proximally, the negative electrode distally. The active recording electrodes are placed on the bellies of the abductor digiti minimi (hypothenar eminence) and adductor pollicis (thenar eminence) muscles. The reference electrodes are placed above the first interphalangeal joint of the little finger and the thumb (tendon insertion sites).

The stimulating ECG electrodes were placed on the lightly abraded alcohol-cleansed skin over the ulnar nerve of the randomized volar forearm near the wrist. The negative (distal) electrode was placed on the ulnar side of the volar forearm, $2 \mathrm{~cm}-3 \mathrm{~cm}$ proximal to the flexor crease. The positive electrode was placed with its center $3 \mathrm{~cm}$ proximally to the negative electrode. The recording electrodes were placed (after proper skin cleaning with alcohol and abrasion) on the thenar and the hypothenar eminence (on the muscle belly of the mAP and $\mathrm{mADM}$, respectively) and reference electrodes on the first interphalangeal joints (muscle insertion) of the thumb and the fifth finger, respectively (Figure 2).

The stimulation protocols (ST and TOF) were identical for the two muscles. The stimulating parameters of ST stimulation were $1 \mathrm{~Hz}$ frequency, $0.2 \mathrm{msec}$ pulse width and $10-60 \mathrm{~mA}$ current intensity that was increased in $10 \mathrm{~mA}$ steps. The stimulating parameters of TOF stimulations were $0.2 \mathrm{msec}$ pulse width, $20 \mathrm{sec}$ interval time between TOF sequences, and 10-60 mA current intensity levels, increased in 10 $\mathrm{mA}$ steps. All ST and TOF stimulations were repeated three times at each current intensity level.

\section{All measurements were saved to the built-in SD card of the device for off-line analysis}

The lowest current intensity of any stimulation mode that elicited a repeatable visible muscle contraction (twitch) in the fingers was considered the clinical threshold current intensity $\left(\mathrm{I}_{\text {Th-clinical }}\right)$ and was recorded on the data sheet. This was compared to the lowest current intensity that could elicit a measurable CMAP $\left(\mathrm{I}_{\mathrm{Th}-\mathrm{EMG}}\right)$. The current intensities that elicited the highest CMAP amplitudes $\left(\mathrm{I}_{\mathrm{Max}}\right)$ were noted.

The volunteers were asked to rate the discomfort of neurostimulation at every current intensity of each stimulation mode on a $0-10$ visual analogue scoring (VAS) scale. Zero represented "no discomfort" and 10 represented "worst pain ever experienced" elicited by the stimulation. Whenever the VAS score exceeded 6 points, the volunteers were asked if they gave their consent to continue the measurement. No volunteer was asked to undergo neurostimulation if he/she felt it would be intolerable.

\section{Study objectives and endpoints}

The primary objective of the study was to provide performance data of the Acacia Designs BV NEAT prototype monitoring device in volunteers and assess the subjective discomfort associated with neurostimulation.

\section{Primary endpoint of the study:}

- Assessment of the ability of the prototype to deliver neurostimulation, and assessment of the ability to acquire muscle action potentials, analyze and record these evoked responses on the SD-card.

\section{Secondary endpoints included:}

- Confirmation that the prototype can independently stimulate and record repetitive patterns of neurostimulation, including $1 \mathrm{~Hz} \mathrm{ST}$ and TOF stimulation protocols;

- Verification of the delivery of neurostimulation at varying current intensities, from the lowest current intensity that produces an evoked response (threshold current, $\mathrm{I}_{\mathrm{Th}-\mathrm{EMG}}$ ) to the current intensity above which the evoked response no longer increases (maximal current, $\mathrm{I}_{\text {Max }}$ ), at increasing current levels separated by steps of $10 \mathrm{~mA}$;

- Examination of the intracurrent variability of CMAP amplitudes evoked by 10-60 mA ST stimulation (difference of highest and lowest CMAP amplitudes at the given intensity). Determine any difference in recording performance between the two hand muscles, two sexes, and dominant $v s$. non-dominant hand; 
Citation: Nemes R, Diószeghy B, Pongrácz A, Tassonyi E, Fülesdi B (2018) Performance Assessment of a New Electromyography-based Neuromuscular Monitor and Subjective Discomfort in Unmedicated Volunteers. J Anesth Clin Res 9: 848. doi: $10.4172 / 2155-6148.1000848$

Page 4 of 8

- Validation of the consistency of TOF stimulations and their deviation from the ideal 1.0 value in unmedicated volunteers. Determine any difference in recording performance between the two hand muscles (mAP and mADM), two sexes, and dominant or nondominant hand;

- Establishment of discomfort, if any, associated with nerve stimulation in the awake, unmedicated volunteers. A visual analogue scoring scale, anchored with 0 (representing no distress) and 10 (representing the worst pain ever experienced) was used to rate the level of discomfort;

- Assessment of skin reactions to the stimulation or recording electrodes, if any.

\section{Data analysis}

All measurements were saved on the internal SD card of the device for post hoc analysis. MATLAB TetraAnalyzerViewer2014a64 software (MathWorks, Natick, MA, USA) was used to measure CMAPs and TOF ratios.

When the parametric assumptions of normality and equal variance were met, paired T-test was used to compare data pairs from the same volunteer and Student's T-test and one-way ANOVA to compare study groups. When the above assumptions were not met, paired Signed Rank test, Mann-Whitney U-test and One-way ANOVA on Ranks were used. For normally distributed variables, mean \pm standard deviation (SD) and for non-normally distributed variables, median and the interquartile range (IQR) are presented. The predetermined level of significance was $\mathrm{p}<0.05$. Sigma-Plot for Windows Version 11.0 (Systat Software Inc., San Jose, California, USA) was used for calculations.

\section{Results}

\section{Demographic data}

Demographic data of the volunteers are summarized in Table 1. There was no statistical difference in age $(\mathrm{p}=0.592)$, body mass index $(\mathrm{p}=0.231)$ and handedness $(\mathrm{p}=1.0)$ among the four groups. All volunteers were right-handed, therefore the terms dominant and nondominant side refer to all volunteers. The female and male groups had similar body weight $(\mathrm{p}=0.145)$, height $(\mathrm{p}=0.684)$ and wrist circumference $(\mathrm{p}=0.247)$.

\begin{tabular}{|c|c|c|c|c|c|}
\hline & $\begin{array}{l}\text { Male } \\
\text { Left hand }\end{array}$ & $\begin{array}{l}\text { Male } \\
\text { Right hand }\end{array}$ & $\begin{array}{l}\text { Female } \\
\text { Left hand }\end{array}$ & $\begin{array}{l}\text { Female } \\
\text { Right hand }\end{array}$ & $\mathrm{p}$ \\
\hline $\begin{array}{l}\text { Age (years) } \\
\text { median (IQR) }\end{array}$ & $23(22.75-30.25)$ & $24(23.75-33)$ & $24(22.75-44.25)$ & $23(21.75-28)$ & 0.59 \\
\hline $\begin{array}{l}\mathrm{BMI}\left(\mathrm{kg} / \mathrm{m}^{2}\right) \\
\text { mean }(\mathrm{SD})\end{array}$ & $25.04(3.43)$ & $22.72(3.19)$ & $26.48(6.89)$ & $21.05(1.95)$ & 0.23 \\
\hline $\begin{array}{l}\text { Body weight }(\mathrm{kg}) \\
\text { mean }(\mathrm{SD})\end{array}$ & $79.6(9.81)^{\star}$ & $71.6(12.92)^{\star}$ & $73.8(16.86) \dagger$ & $60.4(7.8) \dagger$ & $\begin{array}{l}* 0.30 \\
\dagger 0.15\end{array}$ \\
\hline $\begin{array}{l}\text { Height (cm) } \\
\text { mean (SD) }\end{array}$ & $178.4(4.39)^{*}$ & $177.2(8.2)^{\star}$ & $167.6(4.83) \dagger$ & $169.2(6.98) \dagger$ & $\begin{array}{l}{ }^{*} 0.78 \\
\dagger 0.68\end{array}$ \\
\hline $\begin{array}{l}\text { Wrist circumference }(\mathrm{mm}) \\
\text { mean }(\mathrm{SD})\end{array}$ & $176.2(6.18)^{*}$ & $171.2(8.9)^{*}$ & $164.2(12.38) \dagger$ & $156.2(6.54) \dagger$ & $\begin{array}{l}{ }^{*} 0.33 \\
\dagger 0.25\end{array}$ \\
\hline Left:Right-handed & $0: 5$ & $0: 5$ & $0: 5$ & $0: 5$ & $1: 0$ \\
\hline
\end{tabular}

Table 1: Demographic data of the volunteers. Statistical comparison of age and BMI was performed for all study groups. Body weight, height and wrist circumference were statistically compared between the same sex groups. ${ }^{*}$ represents the $\mathrm{p}$ value for the male groups, $\dagger$ represents the $\mathrm{p}$ value for the female volunteers.

\section{Threshold stimulating current intensity}

The threshold current intensity that could elicit visible muscle twitches $\left(\mathrm{I}_{\text {Th-clinical }}\right)$ and recordable CMAPs $\left(\mathrm{I}_{\mathrm{Th}-\mathrm{EMG}}\right)$ was found to be $20(20-30) \mathrm{mA}$ \{median (IQR)\}. The clinical examination $\left(\mathrm{I}_{\mathrm{Th} \text {-clinical }}\right)$ and monitor $\left(\mathrm{I}_{\mathrm{Th}-\mathrm{EMG}}\right)$ recordings were congruent $(\mathrm{p}=0.854)$ in 64 out of 80 stimulation protocols $(80 \%)$. Women needed significantly lower current intensity than men to elicit recordable CMAPs $\{20$ (20-20) vs. $30(20-30) \mathrm{mA}, \mathrm{p}=0.002\}$. The abductor digiti minimi muscle had lower $\mathrm{I}_{\text {Th-EMG }}$ than the adductor pollicis $\{23.75 \pm 7.0$ vs. $25.5 \pm 6.78$ $\mathrm{mA}$ (mean $\pm \mathrm{SD}), \mathrm{p}=0.039)\}$. There was a moderate correlation between wrist circumference and $\mathrm{I}_{\mathrm{Th}-\mathrm{EMG}}$ (Pearson's $\mathrm{r}=0.51, \mathrm{p}=0.0217$ ) and between BMI and $\mathrm{I}_{\mathrm{Th}-\mathrm{EMG}}$ (Pearson's $\mathrm{r}=0.505, \mathrm{p}=0.023$ ).

\section{CMAP amplitude tendencies at increasing intensity ST stimulation}

Increasing current intensities resulted in the increase of elicited CMAP amplitudes (Figure 3). The current intensity that induced the highest CMAPs $\left(\mathrm{I}_{\mathrm{Max}}\right)$ was significantly different between the two muscles. The mADM needed lower current intensity than $\mathrm{mAP}$ to reach maximal stimulation $\{30(20-40) \mathrm{mA}$ vs. 50 (50-60) $\mathrm{mA}$, respectively, $\mathrm{p}<0.001\}$. During maximal stimulation, the highest elicited CMAP amplitudes were moderately lower in the $\mathrm{mADM}$ $(10.40 \pm 1.9 \mathrm{mV})$ than in the $\mathrm{mAP}(12.84 \pm 5.1 \mathrm{mV}), \mathrm{p}=0.031$. 
Citation: Nemes R, Diószeghy B, Pongrácz A, Tassonyi E, Fülesdi B (2018) Performance Assessment of a New Electromyography-based Neuromuscular Monitor and Subjective Discomfort in Unmedicated Volunteers. J Anesth Clin Res 9: 848. doi: $10.4172 / 2155-6148.1000848$

Page 5 of 8
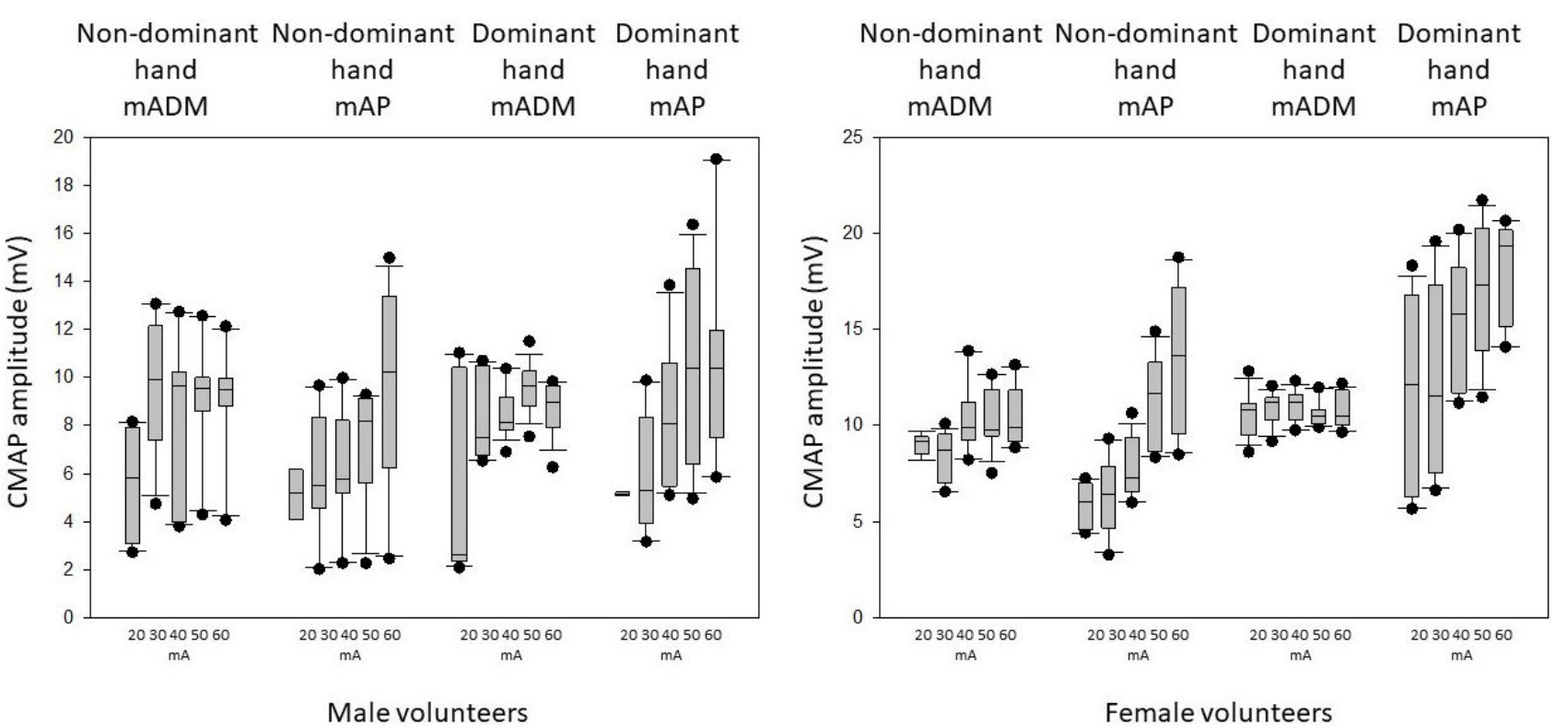

Figure 3: Compound muscle action potentials evoked by increasing intensity stimulation in the two muscles. $\mathrm{mADM}=\mathrm{abductor}$ digiti minimi muscle, $\mathrm{mAP}=$ adductor pollicis muscle. The boxplots represent median with interquartile ranges, the error bars represent $10^{\text {th }}$ and $90^{\text {th }}$ percentiles and the dots represent minimum and maximum values. Boxplots were created in Sigma-Plot for Windows Version 11.0 (Systat Software Inc., San Jose, California, USA).

Female volunteers had higher CMAP amplitudes than men at the same stimulating current intensity, especially in the dominant mAP. Also, the female volunteers required lower stimulating current intensity to elicit maximal CMAP amplitudes than men $\{40$ (20-50) vs. 45 (30-60) $\mathrm{mA}$, respectively, $\mathrm{p}=0.042\}$.

\section{Consistency of CMAP amplitudes (intracurrent differences) in ST protocol at increasing stimulation current}

The consistency of CMAP amplitudes was examined in 240 ST stimulation protocols $(20$ volunteers $\times 2$ muscles $\times 6$ current intensities). Of these, 59 stimulation protocols were excluded from the analysis because the current was under the threshold to evoke measurable CMAP. Another 4 protocols were missed because two female volunteers wished to discontinue the testing at higher (50-60 $\mathrm{mA}$ ) current intensity. Altogether, CMAP amplitude consistency was examined in 177 cases (73.75\%). The overall intracurrent difference, when the results of all stimulating intensities were pooled, was 0.42 (0.21-0.87) $\mathrm{mV}$. In $145 / 177(81.92 \%)$ of cases, the intracurrent difference was $<1.0 \mathrm{mV}$ (Figure 4). There was no statistical difference in the consistency of CMAP amplitudes between sexes \{men: 0.37 (0.18-0.84) $\mathrm{mV}$ vs. women: $0.47(0.25-0.87 \mathrm{mV}, \mathrm{p}=0.285\}$ or between the two muscles (mADM: $0.42(0.24-0.90)$ vs. mAP: $0.44(0.19-0.87)$ $\mathrm{mV}$, respectively, $\mathrm{p}=0.511\}$. The stimulating current intensity $(20,30$, $40,50,60 \mathrm{~mA}$ ) did not influence the level of intracurrent CMAP amplitude variability during ST stimulation $\{0.36(0.24-1.13), 0.28$ (0.20-1.01), 0.33 (0.17-0.61), 0.67 (0.21-0.89), 0.30 (0.15-0.83), respectively, $\mathrm{p}=0.688\}$, if it was high enough to evoke reproducible CMAPs.

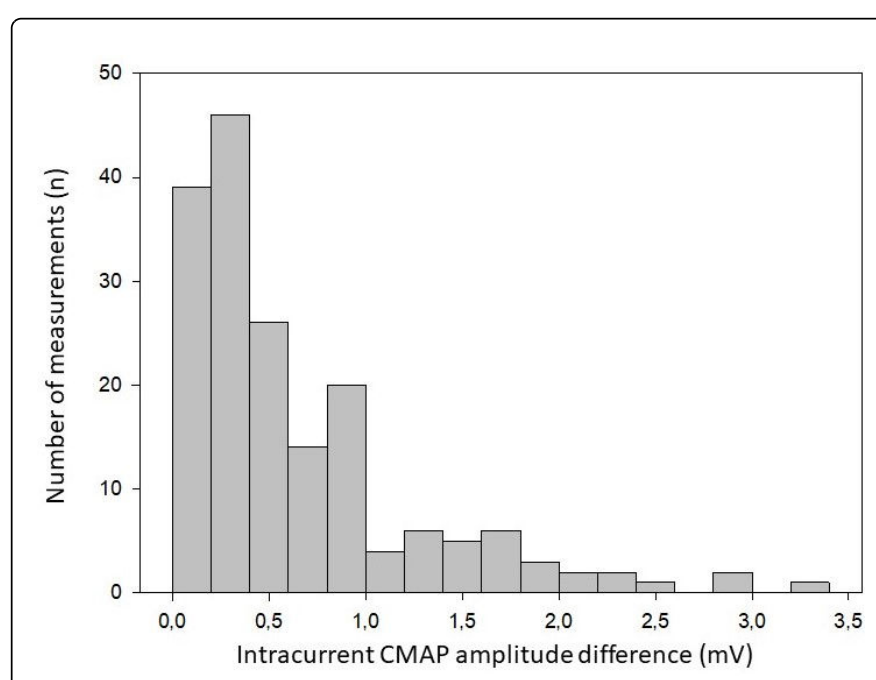

Figure 4: Overall intracurrent compound muscle action potential (CMAP) amplitude variability during single twitch (ST) stimulation. The histogram was created in Sigma-Plot for Windows Version 11.0 (Systat Software Inc., San Jose, California, USA).

\section{Consistency of TOF measurements}

Seven hundred-twenty TOF measurements were performed in the study $(20$ volunteers $\times 2$ muscles $\times 6$ current intensities $\times 3$ stimulations at each current intensity). TOF analysis could be performed in $532(74 \%)$ cases, those in which the stimulating current intensity was sufficient to elicit CMAPs and produce TOF ratios. Two 
Citation: Nemes R, Diószeghy B, Pongrácz A, Tassonyi E, Fülesdi B (2018) Performance Assessment of a New Electromyography-based Neuromuscular Monitor and Subjective Discomfort in Unmedicated Volunteers. J Anesth Clin Res 9: 848. doi: $10.4172 / 2155-6148.1000848$

Page 6 of 8

female volunteers declined TOF stimulation at $50 \mathrm{~mA}$ and $60 \mathrm{~mA}$. In 4 cases, false detection was experienced, as only a TOF count was determined instead of TOF ratio. The overall consistency of elicited TOF ratios was $1.02(0.98-1.06)$. In $445 / 532(83.64 \%)$ of cases, the TOF ratios were in the range of $0.9-1.1$ (Figure 5 ). The TOF ratios were closer to the expected 1.0 in men than in women $\{1.01(0.97-1.06) \mathrm{vs}$. 1.02 (0.99-1.05), respectively, $\mathrm{p}=0.029\}$ and in mADM than in mAP $\{1.01(0.98-1.05)$ vs. $1.02(0.99-1.06)$, respectively, $\mathrm{p}=0.031\}$. The stimulating current intensity $(20,30,40,50,60 \mathrm{~mA})$ did not influence the precision of TOF ratio measurement $\{1.03(0.99-1.06), 1.02$ (0.99-1.07), 1.01 (0.97-1.05), 1.01 (0.98-1.06), 1.02 (0.99-1.05), respectively, $\mathrm{p}=0.256\}$.

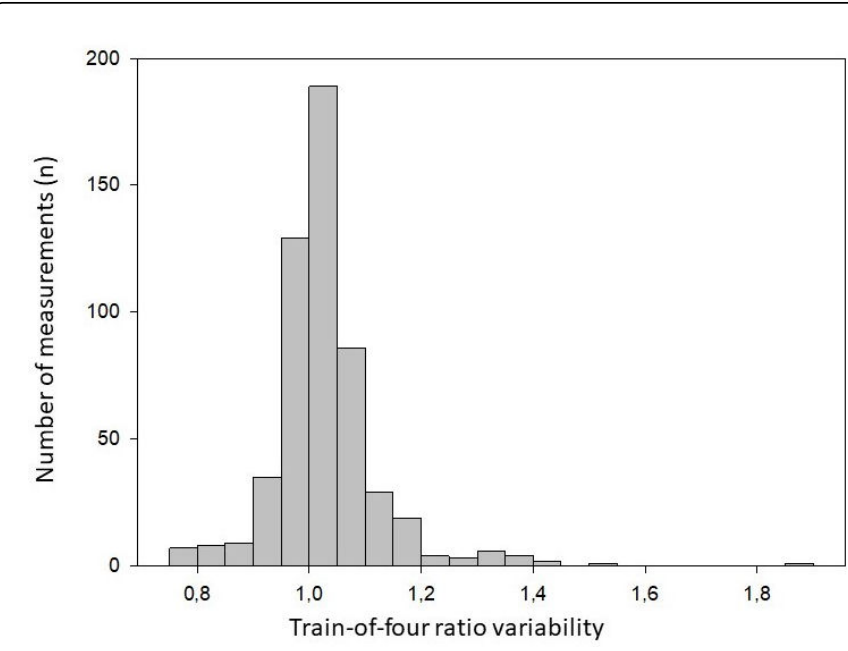

Figure 5: Overall train-of-four ratio variability. The histogram was created in Sigma-Plot for Windows Version 11.0 (Systat Software Inc., San Jose, California, USA).

\section{VAS scores}

Figure 6 shows the VAS scores associated with ST and TOF stimulation protocols at increasing current intensities. The data obtained from the two arms and two muscles of both sex groups were pooled because they did not show statistical difference. The level of discomfort and the corresponding VAS scores increased as a function of increasing stimulating current intensity for both ST and TOF stimulation. Women had higher VAS scores than men at the same current intensities. The largest recorded difference in VAS scores was 5 (3.75-6) vs. 3 (3-4), respectively, at $60 \mathrm{~mA}$ TOF stimulation, $(\mathrm{p}=0.01)$. Two female volunteers refused ST and TOF stimulation at 50 and 60 $\mathrm{mA}$, due to discomfort at $40 \mathrm{~mA}$. The other eighteen volunteers tolerated all stimulation current intensities well. $\mathrm{I}_{\mathrm{Max}}$ stimulating current intensity was associated with a median VAS score of $3(2-5)$ in the pooled cohort.

\section{Skin irritation}

Two volunteers presented mild, painless hyperemia at the stimulation site after removing the ECG electrodes. The hyperemia disappeared in a few minutes and no further irritation or adverse events were reported.

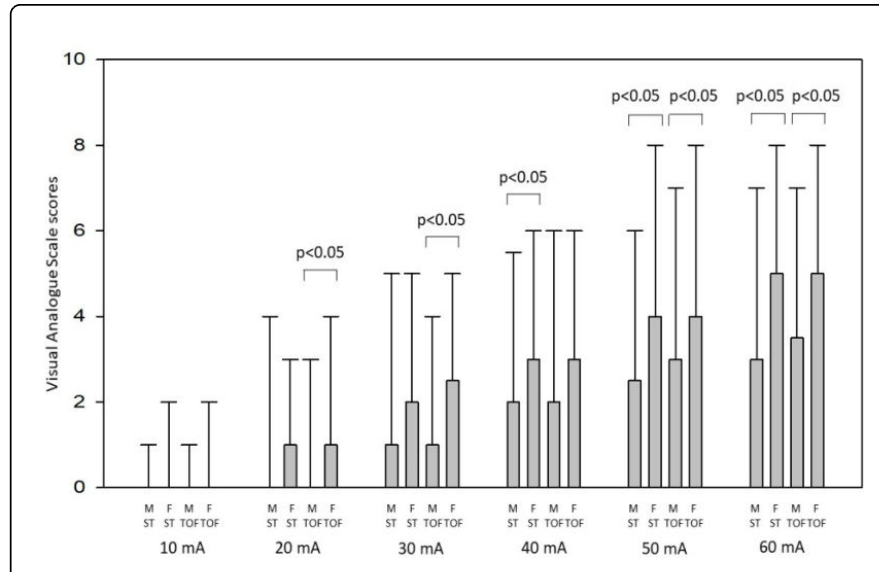

Figure 6: Visual analogue scale scores as a function of increasing intensity single twitch and train-of-four stimulation. Bars represent median values; error bars represent maximal values. Data of leftand right-hand groups of volunteers of the same sex and the two muscles are pooled. M-male groups, F-female groups. The boxplot was created in Sigma-Plot for Windows Version 11.0 (Systat Software Inc., San Jose, California, USA).

\section{Discussion}

The aim of this preclinical investigation was to obtain performance data on the prototype of a new EMG-based neuromuscular monitor. The Acacia Designs BV NEAT monitoring device was able to deliver neurostimulation, record and analyse the elicited muscle action potentials. The maximal CMAP amplitudes of mADM due to ulnar nerve stimulation corresponded to previously reported database normal values [13].

Kopman and Paulus have shown that the current required to produce a detectable muscle twitch via cutaneous electrodes is 5-25 $\mathrm{mA}$, while the current required for supramaximal stimulation is $30-60$ $\mathrm{mA}$ [14-16]. Our results are comparable with these previous reports.

However, current intensity (in $\mathrm{mA}$ ) is not the sole factor that determines the strength (charge) of neurostimulation. The actual charge (in microcoulombs, $\mu \mathrm{C}$ ) that is delivered to the stimulated nerve is the product of the current intensity (in $\mathrm{mA}$ ) and the pulse width (in msec). In general, charges of $12-15 \mu \mathrm{C}$ are required for a maximal muscle response, although some muscles have even higher requirements $[2,17]$. In this equation, the more influential factor is pulse duration. Decreasing the pulse width from $0.2 \mathrm{msec}$ to $0.1 \mathrm{msec}$ (a 50\% reduction) would require a doubling of the stimulating current intensity (from $60 \mathrm{~mA}$ to $120 \mathrm{~mA}$ ) in order to maintain the stimulation charge constant. However, using such high current intensities could be extremely painful to the patients. In this study, 6 volunteers rated the discomfort associated with $60 \mathrm{~mA}$ stimulations more than 6 points on the 0-10 VAS scale. However, the stimulating current intensity necessary for supramaximal stimulation is between $0-70 \mathrm{~mA}$ with a pulse duration of $0.2-0.3 \mathrm{msec}[1,3]$. These parameters can ensure the delivery of supramaximal stimulation without causing direct muscle stimulation that might be elicited by pulse durations in excess of 3-400 msec [2]. The NEAT device meets these requirements as it can deliver ST and TOF stimulation at currents of $10-60 \mathrm{~mA}$ in $10 \mathrm{~mA}$ increments with a $0.2-0.3 \mathrm{msec}$ pulse width. 
Citation: Nemes R, Diószeghy B, Pongrácz A, Tassonyi E, Fülesdi B (2018) Performance Assessment of a New Electromyography-based Neuromuscular Monitor and Subjective Discomfort in Unmedicated Volunteers. J Anesth Clin Res 9: 848. doi: $10.4172 / 2155-6148.1000848$

Page 7 of 8

We aimed to obtain performance data in various subjects; therefore, the demographic characteristics of the volunteers were not standardized. The male and female volunteers showed a difference regarding threshold current $\left(\mathrm{I}_{\mathrm{Th}}\right)$, but the sex of volunteers did not influence the reproducibility (consistency) of ST stimulations. In this cohort, women required lower stimulating currents to elicit recordable CMAPs, and similar intensity stimulation produced higher muscle responses (Figure 3). It is presumed that female volunteers had lower skin impedance. This could have resulted in higher total stimulus charges delivered to the ulnar nerve and higher muscle action potentials. This is consistent with the slightly higher VAS scores reported by female volunteers compared with their male counterparts. However, there was only moderate correlation between BMI or wrist circumference and the threshold current to evoke CMAP as it was previously described by Kopman [14].

Phillips et al. previously studied the evoked electromyographic responses to supramaximal TOF stimulation at the $\mathrm{mAP}$ and $\mathrm{mADM}$ during recovery from non-depolarizing neuromuscular blockade [18]. They found that the mADM was more resistant to NM blockade and recovered faster than $\mathrm{mAP}$. They also concluded that EMG recordings from $\mathrm{mADM}$ were more precise. The repeatability coefficient was lower for $\mathrm{mADM}$ than for $\mathrm{mAP}$ (4.4\% vs. 5.9\%, respectively) [18]. In this study, the mADM required $20 \mathrm{~mA}$ lower stimulating current intensity to reach maximal stimulation $\left(\mathrm{I}_{\mathrm{Max}}\right)$. This resulted in a 1.86point lower VAS scores on the average, hence less discomfort at clinically required maximal stimulation. Additionally, the TOF ratios recorded from $\mathrm{mADM}$ were closer to the expected value of 1.0. This greater consistency of evoked responses and lower stimulating energy (charge) requirement may make the $\mathrm{mADM}$ preferable for monitoring awake patients in the postoperative setting without needing to employ submaximal currents, but this needs further investigation.

Helbo-Hansen et al. studied the repeatability of TOF ratios at varying stimulating currents in anesthetized patients [19]. They found that the accuracy of TOF monitoring was poor at low stimulating currents close to $\mathrm{I}_{\mathrm{Th}}$. Good repeatability was achieved only above 45 $\mathrm{mA}$ stimulating current intensity or $\mathrm{I}_{\mathrm{Th}}+25 \mathrm{~mA}$.

There is a controversy in the literature about the ideal stimulating current for awake patients. Involuntary movement in response to a painful stimulus (withdrawal, contractions) might alter measurements. At the same time, low currents might not deliver equal charges to each stimulus in the TOF sequence. It was previously speculated that submaximal (20-30 mA) stimulation should be used in an awake patient as the best compromise of patient discomfort and repeatability $[16,20]$. However, another study revealed only moderate agreement between two AMG measurements with $30 \mathrm{~mA}$ in the postoperative care unit [21]. In general, our volunteers found the discomfort associated with increasing current intensity acceptable. Our VAS scores were comparable to those in previous reports $[16,17,22]$. In this setting, the stimulating current did not influence the repeatability of TOF measurements; however the study was underpowered for this purpose.

In conclusion, the performance of the electromyography based Acacia Designs BV NEAT device, the prototype of future TetraGraphTM neuromuscular monitor, was acceptable. The present electronics and design would be compatible with the surgical environment. The interface of the device is user-friendly and intuitive. Following further development, the future TetraGraphTM monitor may prove to be an easy to use, reliable and portable NM monitor that fits well in the surgical environment and anesthesia practice.

\section{Declarations}

\section{Ethical approval and consent to participate}

All procedures performed in studies involving human participants were in accordance with the ethical standards of the institutional and the national research committee and with the 1964 Helsinki declaration and its later amendments or comparable ethical standards. The study was approved by the Hungarian Office for Health Authorization and Administrative Procedures on December 30 2014. Identifier: 028605-010/2014/OTIG. Written informed consent was obtained from all individual volunteers prior to inclusion in the study.

\section{Consent for publication}

Not applicable.

\section{Availability of data and material}

The datasets used and/or analysed during the current study are available from the corresponding author on reasonable request.

\section{Competing interests}

Author Réka Nemes has received financial support from Acacia Designs BV (Amsterdam, the Netherlands) to attend the congress of Euroanaesthesia 2015. The other authors declare that they have no conflict of interest.

\section{Funding}

This study was funded by the Hungarian Brain Research Program grant 510 No. KTIA 13 NAPA II/5 and funded by the University of Debrecen Department of Anesthesiology and Intensive Care (Debrecen, Hungary).

\section{References}

1. Lien CA, Kopman AF (2014) Current recommendations for monitoring depth of neuromuscular blockade. Curr Opin Anaesthesiol 27: 616-622.

2. Naguib M, Brull SJ, Johnson KB (2017) Conceptual and technical insights into the basis of neuromuscular monitoring. Anaesthesia 1: 16-37.

3. Brull SJ, Kopman AF (2017) Current status of neuromuscular reversal and monitoring: Challenges and opportunities. Anesthesiology 126: 173-190.

4. Murphy GS, Szokol JW, Avram MJ, Greenberg SB, Marymont JH, et al. (2011) Intraoperative acceleromyography monitoring reduces symptoms of muscle weakness and improves quality of recovery in the early postoperative period. Anesthesiology 115: 946-954.

5. Baillard C (2009) Incidence and complications of postoperative residual paralysis. Ann Fr Anesth Reanim 2: 41-45.

6. Murphy GS, Brull SJ (2010) Residual neuromuscular block: lessons unlearned. Part I: definitions, incidence, and adverse physiologic effects of residual neuromuscular block. Anesth Analg 111: 120-128.

7. Brull SJ, Murphy GS (2010) Residual neuromuscular block: lessons unlearned. Part II: Methods to reduce the risk of residual weakness. Anesth Analg 111: 129-140.

8. Milne JI, Ong CS, Ong JS, Cheung KC, Schauer AA, et al. (2016) The influence of introducing unrestricted access to sugammadex and quantitative neuromuscular monitors on the incidence of residual neuromuscular block at a tertiary teaching hospital. An audit of 'real-life'. Anaesth Intensive Care 44: 784.

9. Kopman AF (2009) Neuromuscular monitoring: old issues, new controversies. J Crit Care 24: 11-20. 
Citation: Nemes R, Diószeghy B, Pongrácz A, Tassonyi E, Fülesdi B (2018) Performance Assessment of a New Electromyography-based Neuromuscular Monitor and Subjective Discomfort in Unmedicated Volunteers. J Anesth Clin Res 9: 848. doi: $10.4172 / 2155-6148.1000848$

Page 8 of 8

10. Checketts MR, Alladi R, Ferguson K, Gemmell L, Handy JM, et al. (2016) Recommendations for standards of monitoring during anaesthesia and recovery 2015: Association of Anaesthetists of Great Britain and Ireland. Anaesthesia 71: 85-93.

11. Claudius C, Skovgaard LT, Viby-Mogensen J (2009) Is the performance of acceleromyography improved with preload and normalization? A comparison with mechanomyography. Anesthesiology 110: 1261-1270.

12. Kopman AF, Kumar S, Klewiczka MM, Neuman GG (2001) The staircase phenomenon: implications for monitoring of neuromuscular transmission. Anesthesiology 95: 403-407.

13. Buschbacher RM (1999) Ulnar nerve conduction to the abductor digiti minimi. Am J Phys Med Rehabil 78: 9-14.

14. Kopman AF, Lawson D (1984) Milliamperage requirements for supramaximal stimulation of the ulnar nerve with surface electrodes. Anesthesiology 61: 83-85.

15. Paulus DA (1987) Electrical signals. In: Gravenstein N, Kirby RR, Brown DL. Problems in Anesthesia: Monitoring. Philadelphia.

16. Brull SJ, Ehrenwerth J, Silverman DG (1990) Stimulation with submaximal current for train-of-four monitoring. Anesthesiology 72: 629-632.
17. Brull SJ, Silverman DG (1995) Pulse width, stimulus intensity, electrode placement, and polarity during assessment of neuromuscular block. Anesthesiology 83: 702-709.

18. Phillips S, Stewart PA, Freelander N, Heller G (2012) Comparison of evoked electromyography in three muscles of the hand during recovery from non-depolarising neuromuscular blockade. Anaesth Int Care 40: 690-696.

19. Helbo-Hansen HS, Bang U, Nielsen HK, Skovgaard LT (1992) The accuracy of train-of-four monitoring at varying stimulating currents. Anesthesiology 76: 199-203.

20. Saitoh Y, Nakazawa K, Toyooka H, Amaha K (1995) Optimal stimulating current for train-of-four stimulation in conscious subjects. Can J Anaesth 42: 992-995.

21. Baillard C, Bourdiau S, Le Toumelin P, Ait Kaci F, Riou B, et al. (2004) Assessing residual neuromuscular blockade using acceleromyography can be deceptive in postoperative awake patients. Anesth Analg 98: 854-857.

22. Connelly NR, Silverman DG, O'Connor TH, Brull SJ (1990) Subjective responses to train-of-four and double burst stimulation in awake patients. Anesth Analg 70: 650-653. 